\title{
The association between expansive arterial remodeling detected by high-resolution MRI in carotid artery stenosis and clinical presentation
}

\author{
Kazumichi Yoshida, MD, PhD, ${ }^{1}$ Ryu Fukumitsu, MD, ${ }^{1}$ Yoshitaka Kurosaki, MD, ${ }^{2}$ \\ Takeshi Funaki, MD, ${ }^{1}$ Takayuki Kikuchi, MD, PhD, ${ }^{1}$ Jun C. Takahashi, MD, PhD, ${ }^{1}$ \\ Yasushi Takagi, MD, PhD, ${ }^{1}$ Sen Yamagata, MD, PhD, ${ }^{2}$ and Susumu Miyamoto, MD, PhD' \\ 'Department of Neurosurgery, Kyoto University Graduate School of Medicine, Kyoto; and 'Department of Neurosurgery, \\ Kurashiki Central Hospital, Okayama, Japan
}

\begin{abstract}
OBJECT The purpose of the present study was to investigate the association between carotid artery (CA) expansive remodeling (ER) and symptoms of cerebral ischemia.

METHODS One hundred twenty-two consecutive CAs scheduled for CA endarterectomy (CEA) or CA stent placement (CAS) were retrospectively studied. After excluding 22 CAs (2 were contraindicated for MRI, 8 had near-occlusion, 6 had poor image quality, and 6 had restenosis after CEA or CAS), there were $100 \mathrm{CAs}$ (100 patients) included in the final analysis. The study included 50 symptomatic patients (mean age $73.6 \pm 8.9$ years, 6 women, mean stenosis $68.5 \% \pm$ $21.3 \%$ ) and 50 asymptomatic patients (mean age $72.0 \pm 5.9$ years, 5 women, mean stenosis $79.4 \% \pm 8.85 \%$ ). Expansive remodeling was defined as enlargement of the internal carotid artery (ICA) with outward plaque growth. The ER ratio was calculated by dividing the maximum distance between the lumen and the outer borders of the plaque perpendicular to the axis of the ICA by the maximal luminal diameter of the distal ICA at a region unaffected by atherosclerosis using long-axis, high-resolution MRI.
\end{abstract}

RESULTS The ER ratio of the atherosclerotic CA was significantly greater than that of normal physiological expansion (carotid bulb; $p<0.01$ ). The ER ratio of symptomatic CA stenosis (median 1.94, interquartile range $[I Q R] 1.58-2.23$ ) was significantly greater than that of asymptomatic CA stenosis (median 1.52, IQR 1.34-1.81; $p=0.0001$ ). When the cutoff value of the ER ratio was set to 1.88 , the sensitivity and specificity to detect symptoms were 0.6 and 0.78 , respectively. The ER ratio of symptomatic patients was consistently high regardless of the degree of stenosis.

CONCLUSIONS There was a significant correlation between ER ratio and ischemic symptoms. The ER ratio might be a potential indicator of vulnerable plaque, which requires further validation by prospective observational study of asymptomatic patients.

http://thejns.org/doi/abs/10.3171/2014.12.JNS14185

KEY WORDS atherosclerosis; carotid stenosis; expansive remodeling; magnetic resonance imaging; vulnerable plaque; vascular disorders

$\mathrm{C}$ AROTID artery (CA) stenosis is a major cause of cerebral infarction and can be treated with medical therapy alone or in combination with interventions such as CA endarterectomy (CEA) or CA stent placement (CAS). While the benefits of CEA or CAS for moderateto high-grade CA stenosis have been established in several previous large clinical trials, ${ }^{4,6,9,32}$ multifaceted medical treatment for atherosclerotic vascular disease has improved dramatically in recent years, and the role of CEA/CAS for stroke prevention, particularly in asymptomatic CA stenosis, needs to be redefined based on more accurate stroke risk stratification.

Therefore, accurate stroke risk stratification for future ischemic events using diagnostic imaging is useful for stroke prevention. Accumulating evidence from recent vascular biology studies demonstrates that the qualitative and

ABBREVIATIONS $C A=$ carotid artery; $\mathrm{CAE}=\mathrm{CA}$ endarterectomy; $\mathrm{CAS}=\mathrm{CA}$ stent placement $\mathrm{Cl}=$ confidence interval; $\mathrm{ER}=$ expansive remodeling; $\mathrm{ICA}=$ internal $\mathrm{CA}$; $\mathrm{IQR}=$ interquartile range; NASCET $=$ North American Symptomatic Carotid Endarterectomy Trial; $\mathrm{OR}=$ odds ratio; ROC = receiver operating characteristic.

SUBMITTED February 5, 2014. ACCEPTED December 18, 2014.

INCLUDE WHEN CITING Published online February 13, 2015; DOI: 10.3171/2014.12.JNS14185.

DISCLOSURE The authors report no conflict of interest concerning the materials or methods used in this study or the findings specified in this paper. 
morphological characteristics of plaque influence the risk of ischemic events, ${ }^{22}$ as well as the luminal narrowing of the CA. However, the selection of treatment modalities for patients with CA stenosis is based mostly on degree of stenosis, symptomatic status, and general physical condition of the patients.

The CA can change its geometry in response to the development of atherosclerosis either by negative remodeling or expansive remodeling (ER), and such arterial remodeling has been extensively investigated in the coronary circulation. Expansive remodeling is enlargement of the atherosclerotic artery with outward plaque growth, was first described in a postmortem histological study, and was initially considered a compensatory response that retarded luminal narrowing and prevented ischemic events. ${ }^{12}$ However, subsequent studies with the advent of intravascular ultrasonography demonstrated that compensatory ER may also be associated with plaque instability and increase the risk of ischemic events. ${ }^{2,14,23}$ Matsuo et al. investigated plaque characteristics and arterial remodeling using intravascular ultrasonography in coronary and peripheral arteries such as the carotid, renal, and iliac arteries, and reported that arteries with ER, compared with those with negative remodeling, demonstrated more characteristics of fibroatheroma, which is one of the most significant features of vulnerable plaque regardless of its location..$^{20}$

There have been 2 previous studies that used multidetector CT angiography to investigate the association between ER in the CA and clinical presentation. ${ }^{13,21}$ The purpose of this study was to assess the feasibility of longaxis CA MRI ${ }^{33}$ for quantitative assessment of ER and to evaluate the association between the extent of ER and cerebral ischemic events.

\section{Methods \\ Patient Population}

Based on prospectively collected data for patients with CA stenosis who were scheduled for CEA or CAS at Kurashiki Central Hospital, a total of 122 consecutive CAs in 119 patients were retrospectively analyzed. Inclusion criteria for CEA/CAS included $\geq 70 \%$ asymptomatic CA stenosis and $\geq 50 \%$ symptomatic stenosis. For symptomatic low-grade stenosis $(<50 \%)$, we considered CEA when patients presented with more than 2 recurrences despite maximal medical therapy and no other general problems, and consented to surgical intervention. Two CAs were contraindicated for MRI due to pacemaker implantation, 8 CAs had near-occlusion, 6 CAs had MR images graded as inadequate for diagnosis due to poor image quality, and 6 CAs had restenosis after CEA $(n=3)$ or CAS $(n=3)$. These 22 CAs were excluded and 100 CAs in 100 patients were included in the final analysis. Symptomatic patients were defined as those who experienced amaurosis fugax, a transient ischemic attack, or stroke in the territory of the ipsilateral internal CA (ICA) within 6 months. There were 50 symptomatic patients and 50 asymptomatic patients. The severity of CA stenosis was evaluated by digital subtraction angiography using criteria from the North American Symptomatic Carotid Endarterectomy Trial (NASCET) Collaborators. ${ }^{4}$
Age, sex, percentage of luminal stenosis, and risk factors for ischemic stroke (hypertension, hyperlipidemia, diabetes mellitus, ischemic heart disease, and smoking habit) were analyzed based on symptoms, and only the percentage of luminal stenosis ( $\mathrm{p}=0.0011)$ and method of revascularization $(\mathrm{p}=0.0001)$ were significantly different between symptomatic and asymptomatic patients (Table 1). Hypertension and hyperlipidemia were by definition restricted to those cases using blood pressure-lowering drugs and statins, respectively. Diabetes mellitus was defined as use of insulin or oral glucose inhibitors. Ischemic heart disease was defined as angina or prior myocardial infarction. These patient characteristics are summarized in Table 1.

The local institutional review board of Kurashiki Central Hospital approved this study, and all patients provided written informed consent to participate in all procedures associated with the study.

\section{Imaging Techniques}

A 1.5-T, whole-body MRI machine (Gyroscan Intera, Philips Medical Systems) with an 8-cm surface coil was used to image the CAs. T1-weighted images of sagittal sections parallel to the long axis of the CA at $1-\mathrm{mm}$ intervals were obtained using the double inversion recovery technique to suppress the blood flow signal. The fat suppression method was applied to suppress marked signal hyperintensity due to subcutaneous fat tissue by using the chemical shift-selective fat suppression technique of spectral presaturation with inversion recovery. The parameters of black-blood MRI were as follows: 3D inversion-recovery turbo field echo, field of view $150 \mathrm{~mm}$, matrix $320 \times 512$, TR/TE/TI 10/2.7/500 msec, flip angle $35^{\circ}$, slice thickness $1.6 \mathrm{~mm}$, and reconstructed voxel size $0.29 \times 0.29 \times 0.8 \mathrm{~mm}$.

\section{Definition of ER and Measurement of the ER Ratio}

In this study, ER was defined as enlargement of the ICA with outward plaque growth more than 3-mm thick

TABLE 1. Characteristics of the patients with CA stenosis scheduled for CEA or CAS

\begin{tabular}{lccc}
\hline \multirow{2}{*}{ Variable } & \multicolumn{2}{c}{ Lesions $(\mathrm{n}=50$ each group) } & \\
\cline { 2 - 3 } & $\begin{array}{c}\text { Symptomatic } \\
(\%)\end{array}$ & $\begin{array}{c}\text { Asymptomatic } \\
(\%)\end{array}$ & p Value \\
\hline Mean age \pm SD $(\mathrm{yrs})$ & $73.6 \pm 8.9$ & $72.0 \pm 5.9$ & 0.3173 \\
\hline Females & $6(12)$ & $5(10)$ & 0.7493 \\
\hline Mean \% stenosis \pm SD $^{*}$ & $68.5 \pm 21.3$ & $79.4 \pm 8.85$ & $0.0011 \dagger$ \\
\hline CEA & $34(68)$ & $15(30)$ & $0.0001 \dagger$ \\
\hline CAS & $16(32)$ & $35(70)$ & $0.0001 \dagger$ \\
\hline Hypertension & $44(88)$ & $44(88)$ & 1 \\
\hline Hyperlipidemia & $26(52)$ & $30(60)$ & 0.4203 \\
\hline Diabetes & $12(24)$ & $20(40)$ & 0.0863 \\
\hline Ischemic heart disease & $19(38)$ & $23(46)$ & 0.4177 \\
\hline Smoking habit & $23(46)$ & $22(44)$ & 0.8407 \\
\hline
\end{tabular}

* According to NASCET criteria.

$\dagger$ Significant difference between the two groups. 
on long-axis high-resolution MRI. The measurement technique for the ER ratio was developed by applying the method used in NASCET to evaluate stenosis..$^{10}$ The ER ratio in the CA was calculated using the long-axis, blackblood $\mathrm{MRI}^{33}$ and the following formula:

ER ratio $=$ the maximum distance between the lumen and the outer borders of the plaque perpendicular to the axis of the ICA/the maximal luminal diameter of the distal ICA at a region unaffected by atherosclerosis (Fig. 1).

When the plaque with maximal outside diameter and the normal distal ICA with maximal outside diameter could not be observed on the same image, 2 separate longaxis images that included the maximal outside diameter of the affected ICA and the distal unaffected ICA were selected for the calculation. The control ER ratio was measured in the contralateral nonatherosclerotic ICA in patients with unilateral CA stenosis and the following formula was used:

ER ratio (control) $=$ the maximal luminal diameter at the normal bulb/the maximal luminal diameter of the ICA well beyond the bulb.

\section{Image Review}

Expansive remodeling ratio measurements were performed by 2 experienced investigators blinded to the clinical information (R.F. and Y.K.). The interobserver reproducibility of the ER ratio was assessed in 25 consecutive patients by determining the intraclass correlation coefficient, which varies from 0 (no agreement) to 1 (perfect agreement).

\section{Statistical Analysis}

Numerical data are expressed as means \pm standard deviations and were compared using Welch's t-test or the Wilcoxon rank-sum test as appropriate. Categorical data were evaluated by using the chi-square test. Multiple logistic regression analysis was performed to adjust for other predictors of ischemic symptom (age, sex, percentage of stenosis, hypertension, hyperlipidemia, diabetes, ischemic heart disease, and smoking habit). The results are expressed as odds ratios (ORs) with 95\% confidence intervals (CIs). Values of the ER ratio to differentiate between the 2 groups were calculated from receiver operating characteristic (ROC) curves. All data were statistically analyzed using SPSS (version 12.0 for Windows, SPSS Inc.) and probability values $<0.05$ were considered statistically significant.

\section{Results}

\section{Interobserver Agreement}

The intraclass correlation coefficient value was 0.782 (95\% CI 0.449-0.909), which indicated substantial to good interobserver agreement.

\section{Normal Carotid Bulb and ER of Atherosclerotic ICA}

In 25 patients with unilateral CA stenosis, the ER ratio in the affected ICA was $1.68 \pm 0.40$ and significantly greater than the control ER ratio of $1.36 \pm 0.15$ in the nonaffected ICA ( $p<0.01$, Fig. 2).

\section{Expansive Remodeling Ratio and CA Ischemic Symptoms}

The ER ratio in symptomatic patients (median 1.94, interquartile range [IQR] 1.58-2.23) was significantly greater than that in asymptomatic patients (median 1.52, IQR 1.34-1.81, $\mathrm{p}=0.0001$; Fig. 3). In multiple logistic
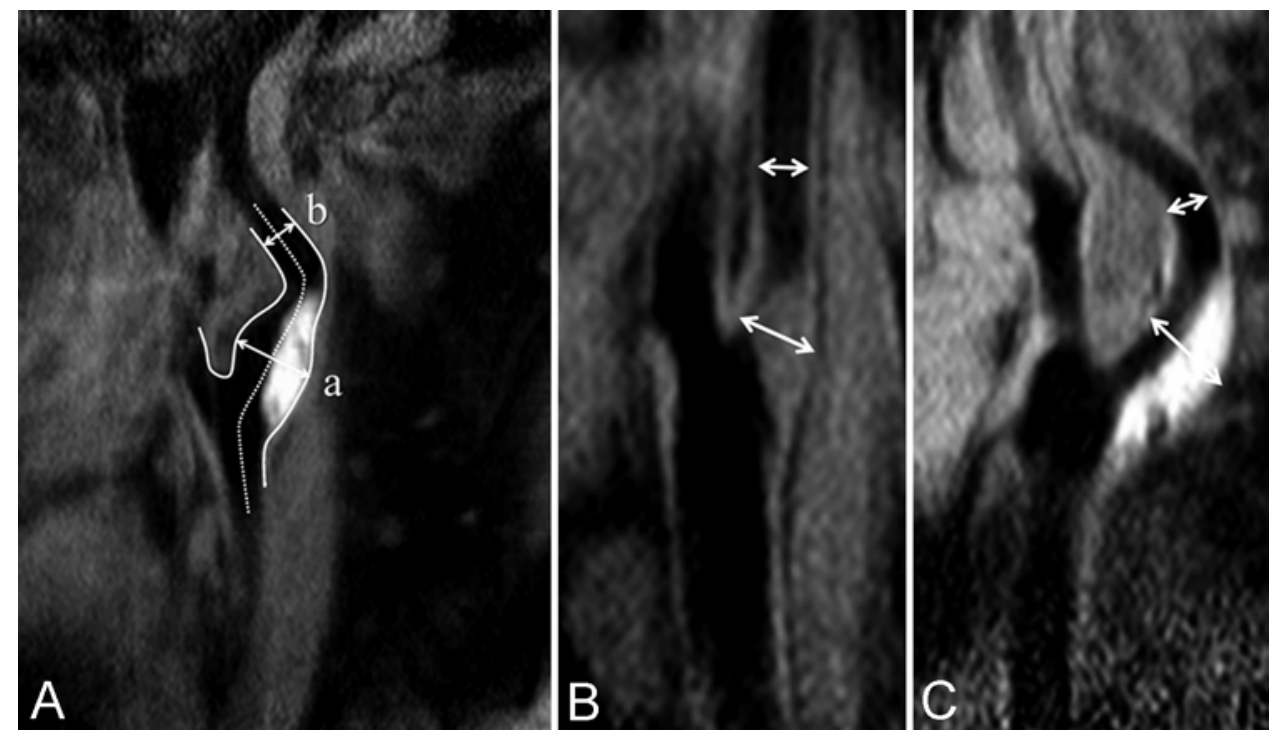

FIG. 1. Long-axis, black-blood MR images. A: Illustration of the measurement of the ER ratio. The ER ratio is calculated as the ratio of the maximum distance between the lumen and the outer borders of the plaque perpendicular to the axis of the ICA (a) and the luminal diameter of the distal ICA (b). The solid lines indicate the luminal surface at a region unaffected by atherosclerosis and the plaque-adventitia interface at an atherosclerotic region. The dotted line indicates the long axis of the ICA. B: Illustrative case of asymptomatic CA stenosis in a 68-year-old male patient. The ER ratio and stenosis percentage were 1.5 and $75 \%$, respectively. C: Illustrative case of symptomatic CA stenosis in a 73-year-old male patient. The ER ratio and stenosis percentage were 2.29 and $20 \%$, respectively. In B and C, the short arrows represent the luminal diameter of the distal ICA, and the long arrows the maximum distance between the lumen and the outer borders of the plaque perpendicular to the axis of the ICA. 


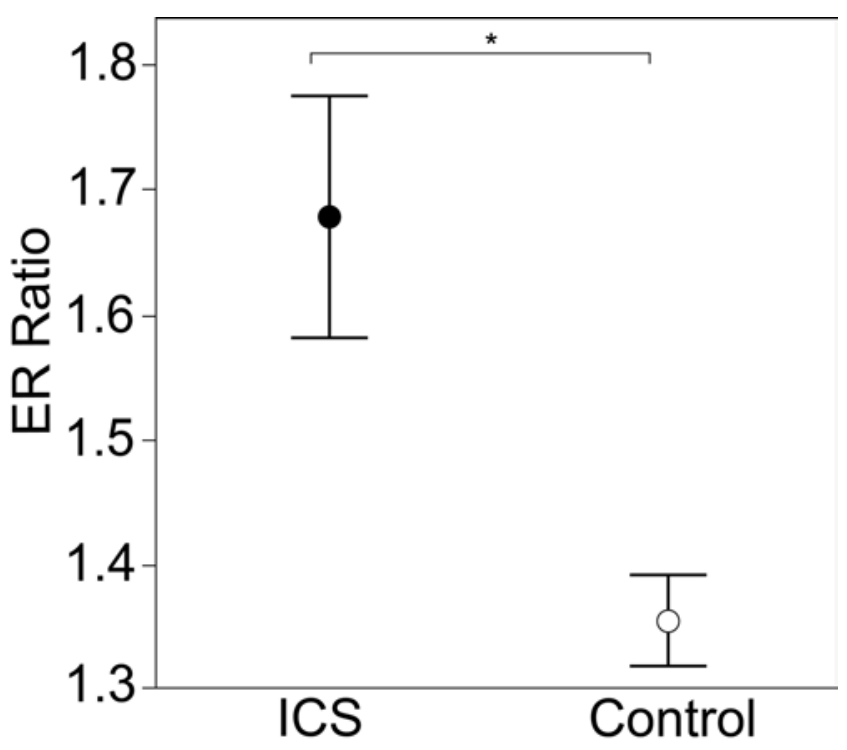

FIG. 2. Comparison between the ER ratio of the atherosclerotic ICA (ICS, or CA stenosis) and the nonatherosclerotic normal ICA (Control) in 25 patients with unilateral $C A$ stenosis. The mean ER ratio in the stenotic ICA $(1.68 \pm 0.40)$ was significantly greater than normal physiological expansion $(1.36 \pm 0.15, p<00.01) .{ }^{*} p<0.01$.

regression analysis, only ER ratio was positively associated with ischemic symptoms (OR $10.683,95 \%$ CI $2.613-$ 43.674; $p=0.001$; Table 2). ROC curve analysis found that the ER ratio could accurately discriminate symptomatic from asymptomatic patients (area under the ROC curve $=0.721$ ). When the cutoff value of the ER ratio was 1.88, the sensitivity and specificity to discriminate symptomatic patients were 0.6 and 0.78 , respectively.

When patients were classified into 4 groups depending on the luminal stenosis rate (CA stenosis $<50 \%, 50 \%-$ $70 \%, 71-80 \%$, and $>81 \%$ ), the mean ER ratios for symptomatic patients were $1.93 \pm 0.54$ (CA stenosis $<50 \%$ ),

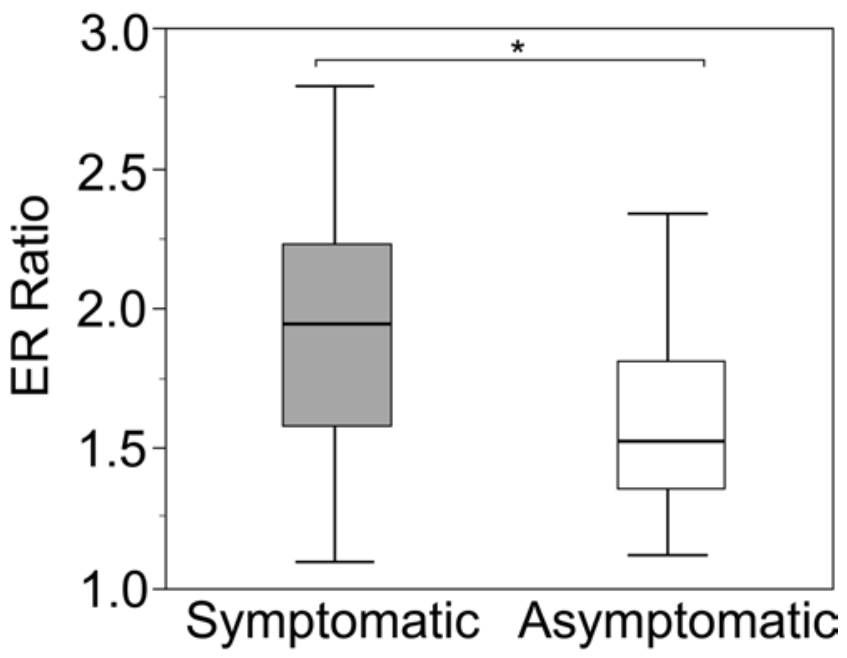

FIG. 3. Comparison of the ER ratio between symptomatic $(n=50)$ and asymptomatic $(n=50)$ patients. The median ER ratio of symptomatic patients (median 1.94, IQR 1.58-2.23) was significantly greater than that of asymptomatic patients (median 1.52, IQR 1.34-1.81; $p=00.0001$ ). ${ }^{*} p$ $<0.001$.
TABLE 2. Multivariate logistic regression analysis of factors associated with ischemic symptoms

\begin{tabular}{lcll}
\hline \multicolumn{1}{c}{ Factor } & OR & \multicolumn{1}{c}{$95 \% \mathrm{Cl}$} & $\mathrm{p} \mathrm{Value}$ \\
\hline ER ratio & 10.683 & $2.613-43.674$ & $0.001^{*}$ \\
\hline Age & 1.033 & $0.967-1.102$ & 6.759 \\
\hline Sex (female) & 1.347 & $0.269-10.301$ & 0.717 \\
\hline \% stenosis & 0.002 & $0.000-0.117$ & $0.003^{*}$ \\
\hline Hypertension & 1.593 & $0.386-6.573$ & 0.520 \\
\hline Hyperlipidemia & 0.687 & $0.242-1.952$ & 0.482 \\
\hline Diabetes & 0.721 & $0.262-1.989$ & 0.528 \\
\hline Ischemic heart disease & 0.742 & $0.279-1.976$ & 0.551 \\
\hline Smoking habit & 1.644 & $0.557-4.849$ & 0.368 \\
\hline
\end{tabular}

* Statistically significant $(p<0.05)$.

$1.93 \pm 0.54(50 \%-70 \%), 1.90 \pm 0.139(71 \%-80 \%)$, and 1.93 $\pm 0.33(>81 \%)$. The ER ratios in symptomatic patients were consistently elevated, regardless of the stenosis rate. In contrast, the mean ER ratios in asymptomatic patients were $1.54 \pm 0.27(50 \%-70 \%), 1.56 \pm 0.37(71 \%-80 \%)$, and $1.67 \pm 0.30(>81 \%)$, and the ER ratio had a tendency to be positively associated with the luminal stenosis rate, although the relationship did not reach statistical significance (Fig. 4).

\section{Discussion}

To our knowledge, this is the first report to confirm the feasibility of MRI to quantitatively assess carotid artery ER and to demonstrate a relationship between the extent of ER and ischemic symptoms. The role of ER in atherosclerotic arterial disease has been extensively investigated

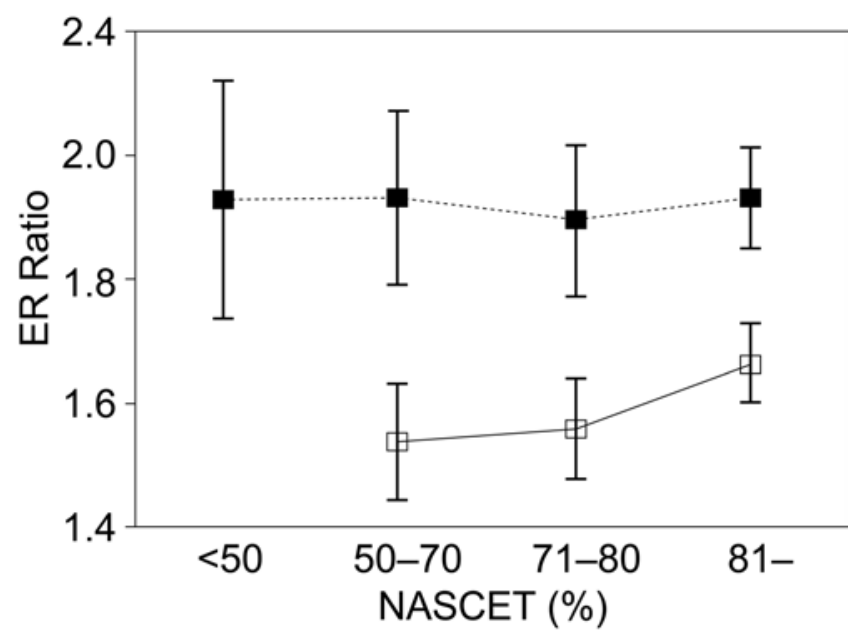

\section{- Symptomatic $\square$ Asymptomatic}

FIG. 4. Expansive remodeling ratios of symptomatic and asymptomatic patients according to the percentage of luminal stenosis of the ICA. The ER ratios for symptomatic patients were $1.93 \pm 0.54$ (CA stenosis $<50 \%), 1.93 \pm 0.54(50 \%-70 \%), 1.90 \pm 0.139(71 \%-80 \%)$, and $1.93 \pm$ $0.33(>81 \%)$, whereas those for asymptomatic patients were $1.54 \pm 0.27$ (50\%-70\%), $1.56 \pm 0.37(71 \%-80 \%)$, and $1.67 \pm 0.30$ (> 81\%). It should be noted that the ER ratio of symptomatic patients was consistently high, regardless of the CA stenosis rate. 
in the coronary circulation, and quantification of ER has been performed predominantly using intravascular ultrasonography. ${ }^{2,14,23}$ However, few studies pertaining to carotid artery ER have been reported and the clinical significance of carotid artery ER remains to be established. ${ }^{13,21}$ Long-axis, high-resolution MRI ${ }^{33}$ was applied to measurement of the carotid artery ER ratio in the present study using the method of stenosis assessment in NASCET, ${ }^{10}$ because a simple, minimally invasive screening modality is needed for the detection of CA vulnerable plaque. There is an inherent difference between coronary artery and CA vasculature, namely, the CA has a normally expanded segment of the carotid bulb, and we first compared the ER ratio of the atherosclerotic ICA with physiological expansion (control ER ratio) in patients with unilateral ICA stenosis and confirmed that the ER ratio of the affected ICA was significantly larger than the control ER ratio (Fig. 2).

The ER ratio in this study was significantly higher in symptomatic than asymptomatic patients, while there was some overlap between them (Fig. 3). Our results agree with those of Hardie et al. ${ }^{13}$ and Miura et al. ${ }^{21}$ who both reported a relationship between ER and symptoms in $\mathrm{CA}$ atherosclerosis evaluated by multidetector CT angiography. Moreover, we demonstrated that the ER ratio in symptomatic patients was consistently elevated regardless of stenosis rate, whereas that of asymptomatic patients tended to increase accordingly with the luminal stenosis rate (Fig. 4). Our data suggest that there is an essential difference in the mechanism for plaque progression when comparing vulnerable with stable plaques, and the ER ratio is more important than the stenosis rate for the early diagnosis of high-risk plaque requiring CA intervention. Postmortem histological studies in the coronary circulation have demonstrated that plaques with ER have a significantly larger lipid core, contain more macrophages, and have an enhanced activity of matrix metalloproteinases compared with negative remodeling plaques. ${ }^{19,24,31}$ Also, in a basic study of macrophage-rich CAs that developed in apolipoprotein E knockout mice, Ivan et al..$^{15}$ found that macrophages facilitate ER through increased matrix degradation by matrix metalloproteinases. Although the precise mechanism responsible for ER remains to be elucidated, the results of these previous studies have consistently shown that there is a close relationship between ER and an inflamed plaque at high risk of rupture.

The benefit of CEA for moderate- to high-grade stenosis in patients with symptomatic and asymptomatic CA stenosis has been established in several previous multicenter trials. ${ }^{4,9,32}$ The effectiveness of CAS has also been established as a useful alternative to CEA in a recent large clinical trial. ${ }^{6}$ However, risk stratification for stroke and the need for CA intervention in these randomized trials was based merely on the percentage of luminal stenosis, whereas recent progress in vascular biology $y^{7,22}$ and the development of vessel wall imaging ${ }^{3,18,26}$ have proven that plaque characteristics and morphology, as well as the severity of stenosis, have a significant influence on the ischemic event rate. For example, Takaya et al. conducted a prospective study in asymptomatic patients with 50\%-79\% CA stenosis and examined the relationship between baseline CA plaque features identified by MRI and the subse- quent CA ischemic events. ${ }^{29}$ This prospective assessment with MRI demonstrated that thinned or ruptured fibrous caps, intraplaque hemorrhage, larger maximum lipid-rich necrotic cores, and larger maximum wall thickness were associated with the development of subsequent ischemic events. In addition to technical innovations for the diagnosis of vascular lesions, multifaceted medical treatment for atherosclerotic vascular disease has improved dramatically in recent years, and this has led some to call for redefining the role of CEA/CAS for stroke prevention in CA stenosis. ${ }^{1}$ Therefore, there is a growing need for accurate detection of truly high-risk patients. The ER ratio in the CA determined by MRI might contribute to more rigorous risk stratification of patients for cerebral ischemic events. As plaque instability is not merely a local vascular occurrence, but also exists simultaneously at multiple sites in the systemic vascular beds, the carotid artery ER ratio could be useful also for predicting other ischemic events in coronary or peripheral arteries.

In addition to MRI, there are other modalities that can be used for quantitative assessment of carotid artery ER, and one of these is catheter-based intravascular ultrasonography. ${ }^{27}$ However, CA ultrasonography, multidetector CT angiography, ${ }^{13,21}$ and MRI are better suited for screening patients for unstable plaque in the clinical setting because they are less invasive. Ultrasonography has many methodological advantages such as noninvasiveness, ease of use, and suitability for repeated imaging. However, for the evaluation of CA circulation, ultrasonography has some limitations. Plaque with dense calcification, a CA with marked meandering, and a stenosis at a high position cannot be fully imaged on CA ultrasonography. The feasibility of CA ultrasonography for assessing the ER ratio should be tested in the future. Compared with multidetector CT angiography, MRI is superior in that it does not deliver radiation ${ }^{5}$ and there are no contrast-induced adverse effects. ${ }^{8,11,25}$ Moreover, MRI could be more accurate in demonstrating the tissue surrounding the vessel wall with high-contrast resolution and measurement of the ER ratio. In addition to the accuracy of carotid artery ER measurement, MRI-which is the only vascular imaging technique capable of detecting the presence and the age of CA intraplaque hemorrhage ${ }^{28}$ - can provide more precise information on the plaque components. Carotid artery ER evaluation in combination with MRI plaque characterization could reliably detect the presence of vulnerable plaque, which is subject to future investigation. On the other hand, MRI has several limitations. First, MRI requires more time for imaging and is more susceptible to motion artifacts. The spatial resolution of MRI is not as good as that of multidetector CT angiography. Second, MRI cannot be performed in patients with medical devices such as a pacemaker, or in patients who cannot lie still, as often occurs with an acute stroke. Third, MRI cannot provide an evaluation of vessel diameter due to poor image quality because of motion artifacts in approximately $5 \%$ of patients. Moreover, although the accuracy of highresolution MRI for CA plaque characterization has been firmly established, ${ }^{30}$ such accuracy has not yet been established for evaluating the vessel outer wall. Expansive remodeling assessment with MRI requires further valida- 
tion by comparison studies with intraoperative vessel wall measurements and preoperative assessment of the ER ratio in patients undergoing CEA. However, the limitations in current MRI diagnosis should be overcome with technological developments such as surface coil design and stronger magnetic fields..$^{16,17}$

Our results have limitations inherent in the retrospective design of the study. First, it is possible that the elevated ER ratios that we found in symptomatic patients could be the result, rather than the cause, of ischemic events. A plaque with a high ER ratio in a symptomatic patient could have merely represented fresh intraplaque hemorrhage. Although this plaque may have been the embolic source, the imaging may only be a representation of previously ruptured plaque, which would undermine the potential predictive value of the ER ratio for ischemic events. Second, our results were based on a comparison between patients known to be symptomatic and those not yet symptomatic, and could not be simply used for the prediction of stroke in a prospective analysis of asymptomatic patients. Studies correlating the histology of plaque excised in CEA with the ER ratio in asymptomatic patients, and more importantly, long-term prospective studies in asymptomatic patients with early-stage CA atherosclerosis, are needed to prove the value of the ER ratio for accurate stroke risk stratification.

\section{Conclusions}

In our series of patients with CA stenosis scheduled for CEA or CAS, the degree of ER in the CA measured with long-axis, high-resolution MRI was significantly greater in symptomatic than asymptomatic patients. Moreover, the elevated ER ratio in symptomatic patients was independent of the severity of stenosis. The ER in atherosclerotic CAs might be an important indicator of vulnerable plaque, which should be validated by prospective observational study for asymptomatic patients.

\section{References}

1. Abbott AL: Medical (nonsurgical) intervention alone is now best for prevention of stroke associated with asymptomatic severe carotid stenosis: results of a systematic review and analysis. Stroke 40:e573-e583, 2009

2. Alfonso F, Macaya C, Goicolea J, Iñiguez A, Hernandez R, Zamorano J, et al: Intravascular ultrasound imaging of angiographically normal coronary segments in patients with coronary artery disease. Am Heart J 127:536-544, 1994

3. Altaf N, Daniels L, Morgan PS, Auer D, MacSweeney ST, Moody AR, et al: Detection of intraplaque hemorrhage by magnetic resonance imaging in symptomatic patients with mild to moderate carotid stenosis predicts recurrent neurological events. J Vasc Surg 47:337-342, 2008

4. Barnett HJ, Taylor DW, Eliasziw M, Fox AJ, Ferguson GG, Haynes RB, et al: Benefit of carotid endarterectomy in patients with symptomatic moderate or severe stenosis. N Engl J Med 339:1415-1425, 1998

5. Brenner DJ, Hall EJ: Computed tomography-an increasing source of radiation exposure. N Engl J Med 357:2277-2284, 2007

6. Brott TG, Hobson RW II, Howard G, Roubin GS, Clark WM, Brooks W, et al: Stenting versus endarterectomy for treatment of carotid-artery stenosis. N Engl J Med 363:11-23, 2010
7. Carr S, Farb A, Pearce WH, Virmani R, Yao JS: Atherosclerotic plaque rupture in symptomatic carotid artery stenosis. J Vasc Surg 23:755-766, 1996

8. Cashman JD, McCredie J, Henry DA: Intravenous contrast media: use and associated mortality. Med J Aust 155:618623,1991

9. European Carotid Surgery Trialists' Collaborative Group: Randomised trial of endarterectomy for recently symptomatic carotid stenosis: final results of the MRC European Carotid Surgery Trial (ECST). Lancet 351:1379-1387, 1998

10. Fox AJ: How to measure carotid stenosis. Radiology 186:316-318, 1993

11. Fradkin JE, Wolff J: Iodide-induced thyrotoxicosis. Medicine (Baltimore) 62:1-20, 1983

12. Glagov S, Weisenberg E, Zarins CK, Stankunavicius R, Kolettis GJ: Compensatory enlargement of human atherosclerotic coronary arteries. N Engl J Med 316:1371-1375, 1987

13. Hardie AD, Kramer CM, Raghavan P, Baskurt E, Nandalur KR: The impact of expansive arterial remodeling on clinical presentation in carotid artery disease: a multidetector CT angiography study. AJNR Am J Neuroradiol 28:1067-1070, 2007

14. Hermiller JB, Tenaglia AN, Kisslo KB, Phillips HR, Bashore TM, Stack RS, et al: In vivo validation of compensatory enlargement of atherosclerotic coronary arteries. Am J Cardiol 71:665-668, 1993

15. Ivan E, Khatri JJ, Johnson C, Magid R, Godin D, Nandi $\mathrm{S}$, et al: Expansive arterial remodeling is associated with increased neointimal macrophage foam cell content: the murine model of macrophage-rich carotid artery lesions. Circulation 105:2686-2691, 2002

16. Koning W, Bluemink JJ, Langenhuizen EA, Raaijmakers AJ, Andreychenko A, van den Berg CA, et al: High-resolution MRI of the carotid arteries using a leaky waveguide transmitter and a high-density receive array at 7 T. Magn Reson Med 69:1186-1193, 2013

17. Kröner ES, Westenberg JJ, van der Geest RJ, Brouwer NJ, Doornbos J, Kooi ME, et al: High field carotid vessel wall imaging: a study on reproducibility. Eur J Radiol 82:680685,2013

18. Kurosaki Y, Yoshida K, Endo H, Chin M, Yamagata S: Association between carotid atherosclerosis plaque with high signal intensity on T1-weighted imaging and subsequent ipsilateral ischemic events. Neurosurgery 68:62-67, 2011

19. Libby P: Current concepts of the pathogenesis of the acute coronary syndromes. Circulation 104:365-372, 2001

20. Matsuo Y, Takumi T, Mathew V, Chung WY, Barsness GW, Rihal CS, et al: Plaque characteristics and arterial remodeling in coronary and peripheral arterial systems. Atherosclerosis 223:365-371, 2012

21. Miura T, Matsukawa N, Sakurai K, Katano H, Ueki Y, Okita $\mathrm{K}$, et al: Plaque vulnerability in internal carotid arteries with positive remodeling. Cerebrovasc Dis Extra 1:54-65, 2011

22. Naghavi M, Libby P, Falk E, Casscells SW, Litovsky S, Rumberger J, et al: From vulnerable plaque to vulnerable patient: a call for new definitions and risk assessment strategies: Part I. Circulation 108:1664-1672, 2003

23. Nakamura M, Nishikawa H, Mukai S, Setsuda M, Nakajima $\mathrm{K}$, Tamada $\mathrm{H}$, et al: Impact of coronary artery remodeling on clinical presentation of coronary artery disease: an intravascular ultrasound study. J Am Coll Cardiol 37:63-69, 2001

24. Pasterkamp G, Schoneveld AH, Hijnen DJ, de Kleijn DP, Teepen H, van der Wal AC, et al: Atherosclerotic arterial remodeling and the localization of macrophages and matrix metalloproteases 1,2 and 9 in the human coronary artery. Atherosclerosis 150:245-253, 2000

25. Rudnick MR, Kesselheim A, Goldfarb S: Contrast-induced nephropathy: how it develops, how to prevent it. Cleve Clin J Med 73:75-80, 83-77, 2006 
26. Saam T, Hatsukami TS, Takaya N, Chu B, Underhill H, Kerwin WS, et al: The vulnerable, or high-risk, atherosclerotic plaque: noninvasive MR imaging for characterization and assessment. Radiology 244:64-77, 2007

27. Siewiorek GM, Loghmanpour NA, Winston BM, Wholey MH, Finol EA: Reproducibility of IVUS border detection for carotid atherosclerotic plaque assessment. Med Eng Phys 34:702-708, 2012

28. Takaya N, Yuan C, Chu B, Saam T, Polissar NL, Jarvik GP, et al: Presence of intraplaque hemorrhage stimulates progression of carotid atherosclerotic plaques: a high-resolution magnetic resonance imaging study. Circulation 111:27682775,2005

29. Takaya N, Yuan C, Chu B, Saam T, Underhill H, Cai J, et al: Association between carotid plaque characteristics and subsequent ischemic cerebrovascular events: a prospective assessment with MRI-initial results. Stroke 37:818-823, 2006

30. Underhill HR, Hatsukami TS, Fayad ZA, Fuster V, Yuan C: MRI of carotid atherosclerosis: clinical implications and future directions. Nat Rev Cardiol 7:165-173, 2010

31. van der Wal AC, Becker AE, van der Loos CM, Das PK: Site of intimal rupture or erosion of thrombosed coronary atherosclerotic plaques is characterized by an inflammatory process irrespective of the dominant plaque morphology. Circulation 89:36-44, 1994

32. Walker MD, Marler JR, Goldstein M, Grady PA, Toole JF,
Baker WH, et al: Endarterectomy for asymptomatic carotid artery stenosis. JAMA 273:1421-1428, 1995

33. Yoshida K, Endo H, Sadamasa N, Narumi O, Chin M, Inoue $\mathrm{K}$, et al: Evaluation of carotid artery atherosclerotic plaque distribution by using long-axis high-resolution black-blood magnetic resonance imaging. J Neurosurg 109:1042-1048, 2008

\section{Author Contributions}

Conception and design: Yoshida. Acquisition of data: Yoshida, Fukumitsu, Kurosaki. Analysis and interpretation of data: Yoshida, Fukumitsu, Kurosaki, Takahashi, Takagi. Drafting the article: Yoshida. Critically revising the article: Fukumitsu, Kurosaki, Funaki, Kikuchi, Takahashi, Takagi, Yamagata. Reviewed submitted version of manuscript: Funaki, Kikuchi, Miyamoto. Approved the final version of the manuscript on behalf of all authors: Yoshida. Statistical analysis: Funaki. Study supervision: Kikuchi, Takahashi, Takagi, Yamagata, Miyamoto.

\section{Correspondence}

Kazumichi Yoshida, Department of Neurosurgery, Kyoto University Graduate School of Medicine, 54 Kawahara-cho, Shogoin, Sakyo-ku, Kyoto 606-8507, Japan. email address: kazuy@kuhp. kyoto-u.ac.jp. 